Código JEL: M3

\title{
Claves del proceso de compra organizacional de vino. El caso de los minoristas en La Rioja
}

\author{
Yolanda SIERRA-MURILLO \\ Universidad de La Rioja \\ yolanda.sierra@unirioja.es \\ Cristina OLARTE-PASCUAL \\ Universidad de La Rioja \\ cristina.olarte@unirioja.es \\ Lidia GIL FERNÁNDEZ \\ Universidad de La Rioja \\ lidia.gil@alumna.unirioja.es
}

Recibido: 10-3-2016

Aceptado: 10-09-2016

\section{RESUMEN}

Junto con las grandes bodegas conviven en el mercado un gran número de pequeñas bodegas, cuyos principales clientes son negocios minoristas. El objetivo del presente trabajo es conocer las claves del proceso de compra organizacional de vino por parte de minoristas del sector HORECA (bares y restaurantes) y distribución especializada (vinotecas). Los resultados reflejan que las compras que realizan estos minoristas se fundamentan sobre una clara orientación al consumidor final por lo que las bodegas deben enfocarse en "los clientes de sus clientes" para conseguir los mejores resultados. A los clientes finales les gusta probar nuevos vinos y valoran una buena relación calidad precio. Las bodegas deberán cuidar la fidelidad de los minoristas con un número de visitas adecuado, sin presionar y con un servicio rápido y puntual. El trabajo concluye con implicaciones para la gestión. 
Palabras clave: vino, compra organizacional de vino, demanda de vino, distribución minorista de vino.

\title{
Key organizational buying process of wine: the case of retailers in La Rioja
}

\begin{abstract}
Along with the great wineries live a large number of small wineries, whose main customers are retail businesses on the market. The aim of this study is to determine the keys of the organizational buying wine process by retailers of the HORECA sector (bars and restaurants) and specialized distribution (wine bars). The results show that purchases by these retailers are based on a clear focus on the end user so that wineries should focus on "customers of its customers" to get the best results. Final customers like to try new wines and appreciate a good value. The wineries should take care of the faithfulness of retailers with an adequate number of visits, without pressing and quick and timely service. The paper concludes with implications for management.
\end{abstract}

Key words: Wine, Organizational buying behavior, wine demand, wine retail.

Sumario: 1. El proceso de compra organizacional. 2. Metodología. 3. Resultados. 4. Recomendaciones y conclusiones.

\section{EI PROCESO DE COMPRA ORGANIZACIONAL}

Junto con las grandes bodegas subsisten gran número de pequeñas bodegas, muchas de ellas de carácter familiar, cuyos clientes principales son pequeñas empresas minoristas del sector HORECA y de la alimentación: restaurantes/bares y vinotecas, respectivamente. El objetivo de este trabajo es analizar el proceso de compra organizacional de vino por parte de dichos minoristas.

Webster y Wind (1972a p. 2) definen la compra organizacional como el proceso de toma de decisiones en el que las organizaciones formales establecen la necesidad de adquirir productos y servicios, e identifican, evalúan y eligen entre las diferentes marcas y proveedores disponibles (Kotler y Keller, 2012; 183). Cada organización tiene objetivos, políticas, procedimientos, estructuras organizacionales y sistemas específicos de compra (Manrique Molina, 2014). En este proceso, el comprador empresarial se enfrenta a numerosas decisiones que van a depender de diferentes factores como el tipo de compra (Robinson, Faris y Wind, 1967): (1) en la compra nueva o compra de un producto o servicio por primera vez, las decisiones requerirán más tiempo y esfuerzo; (2) en la recompra directa o compra de repetición muchas rutinas se repiten y el proceso se simplifica; (3) en la recompra modificada o compra en la que se modifica alguna 
especificación del producto u otros requisitos, se da un proceso intermedio entre los dos anteriores.

Desde un punto de vista histórico, el libro publicado por Robinson, Faris y Wind en el año 1967 junto con los modelos generales propuestos por Webster y Wind (1972a y 1972b) y Sheth (1973) supusieron los inicios conceptuales del estudio del comportamiento de compra organizacional (Johnston y Lewin, 1996). Manrique Molina (2014) sostiene que durante los años ochenta la investigación se desarrolló para entender y medir los diferentes factores que afectan al proceso además de conocer a los actores del proceso y el intercambio de información que se produce (Ej. Fortin y Brent Ritchie, 1980; Moriarty y Spekman, 1984; Ronchetto, Hunt y Reingen, 1989; Johnston y Bonoma, 1981; Johnston y Spekman, 1987). En la década de los noventa la investigación se centra en la relación vendedor comprador industrial (Ej. Wilson, Lilien y Wilson, 1991; Wilson, 1995, Sheth, 1996). En el siglo XXI, Lewin y Donthu (2005) llevan a cabo un meta-análisis de las relaciones entre la naturaleza de la situación de compra, la estructura del centro de compras y la participación de dicho centro de compras. Otra línea se ha centrado en el desarrollo de las nuevas tecnologías y presenta la posibilidad de una convergencia entre los mercados industriales y de consumo (Wind, 2006). Por último, destacar el estudio de la influencia de la marca sobre el centro de compras (ej. Brown, Zablah, Bellenger, y Donthu, 2012).

Gráfico 1. Etapas del proceso de decisión de compra organizacional

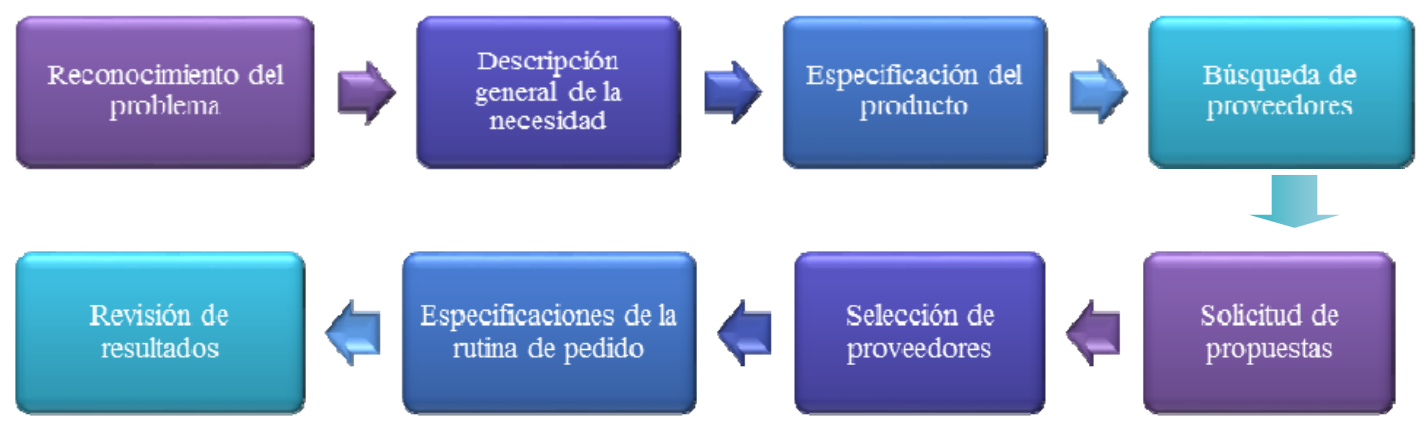

Fuente: elaboración propia a partir de Kotler y Keler (2012).

El proceso de decisión de compra de vino de los minoristas tiene como función obtener una oferta de calidad adecuada para su mercado objetivo. Es decir, necesita identificar la cantidad de bebida necesaria para atender la demanda de sus clientes con una oferta de calidad al mejor precio respaldada por proveedores adecuados que cumplan con los requisitos de utilidad de tiempo y espacio óptimo. El gráfico 1 , recoge una lista de 8 etapas que se identifican en el proceso de compra organizacional cuando se trata de una compra nueva (Kotler y Keler, 2012). En el 
caso de recompra modificada o recompra directa, algunas etapas se omiten o se comprimen:

1. Reconocimiento del problema. El proceso de compra se inicia con el reconocimiento de un problema o necesidad que se puede satisfacer con la adquisición de un bien o servicio. Este reconocimiento del problema se puede suscitar por factores internos, como la necesidad de aumentar la oferta, o por factores externos, como el requerimiento de un nuevo vino por parte de los clientes.

2. Descripción general de la necesidad. A continuación, el comprador determina las características del producto y el número de unidades necesarias para resolver la necesidad. En esta fase, los proveedores (bodegas, por ejemplo) intentarán que sus clientes potenciales (bares, restaurantes o vinotecas, por ejemplo) tengan toda la información posible acerca de sus vinos, de sus características, de forma que ayude al comprador en esta etapa.

3. Especificaciones del producto. La organización compradora determinará las características y especificaciones técnicas del producto. En la compra de vino, la denominación de origen, el tipo de crianza y el envase entre otros, son ejemplos de especificaciones demandadas en el sector.

4. Búsqueda de proveedores. En la cuarta etapa, el comprador intenta identificar a los proveedores más adecuados. Después de evaluar a cada uno de ellos, el comprador termina con una pequeña lista de proveedores cualificados. La tarea de las bodegas en esta etapa es aparecer en aquellos directorios más visitados por sus potenciales clientes y construirse una buena imagen en el mercado.

5. Solicitud de propuestas. El comprador invita a los proveedores seleccionados a hacerles llegar sus propuestas. Las bodegas han de trabajar estas propuestas teniendo en cuenta la cultura de sus clientes. Se han de cuidar los mensajes y argumentos para conseguir el pedido.

6. Selección de proveedores. Para ello, los compradores utilizan una serie de criterios de evaluación como rapidez de entrega, relación calidad-precio, entre otros. A este respecto, las bodegas deben tener la habilidad de identificar cuáles son los criterios de evaluación manejados por sus clientes y potenciales clientes y su evolución en el tiempo.

7. Especificaciones de la rutina de pedido. Después de seleccionar al proveedor, el comprador negocia el pedido final, concretando las especificaciones técnicas, la cantidad de unidades necesarias, el tiempo de entrega, las políticas de devolución y las garantías. La etapa se completa cuando el pedido está en manos del comprador y este puede servirlo. El CRP (continuos replenishment o reaprovisionamiento continuo) agiliza esta etapa.

8. Revisión de resultados. Por último, el comprador revisa periódicamente el desempeño de los proveedores y la calidad del producto. Dependiendo de su 
satisfacción, continuará o no con la relación. La tarea de la bodega, como proveedor, será revisar su desempeño para asegurarse que está proporcionando la satisfacción esperada.

En las líneas anteriores se constata que las organizaciones cuando compran se enfrentan a numerosas decisiones. El proceso de compra varía dependiendo del tipo de productos (Kraljic, 1993), de la complejidad del problema que se esté resolviendo, de lo novedoso que sea el requerimiento de compra, de la cantidad de personas involucradas y del tiempo disponible.

Con este trabajo se pretende aportar información sobre cómo unas organizaciones minoristas toman sus decisiones de compra de vino. En concreto, se pretende dar respuesta a las siguientes preguntas:

- ¿Qué cambios han percibido en la demanda de vino del consumidor final y en la oferta que reciben de las bodegas?

- ¿Qué fuentes de información utilizan?

- ¿Cuáles son los aspectos más valorados para seleccionar un vino?

- ¿Cuáles son las características más valoradas de un proveedor de vino?

- ¿Cómo realizan los pedidos? ¿A qué precios están comprando? ¿Son fieles a los proveedores?

Conociendo las respuestas, las bodegas estarán en disposición de mejorar la satisfacción de sus clientes y sus ventas. El interés del estudio radica en que los procesos de compra y demanda de productos y servicios industriales difiere enormemente del comportamiento que se presenta en los mercados de bienes de consumo (Honnalli, 2011). Este estudio puede contribuir a impulsar la demanda del sector vitivinícola y abrir esta línea de investigación para mejorar el conocimiento de las compras organizacionales en dicho sector.

\section{METODOLOGÍA}

La estructura empresarial del sector vitivinícola se caracteriza por una elevada atomización de las empresas y elevado número de marcas existentes, en muchos casos, de baja notoriedad (Fuentes-Lombardo, Fernández-Ortiz y Cano-Rubio, 2011). Respecto a los canales de distribución, dos tercios del vino que se vende en nuestro país se distribuyen a través de los canales de alimentación y hostelería (Nielsen 2014). En el caso de La Rioja, el canal de distribución principal es HORECA (Barco, Navarro y Pinillos, 2007). Dentro del canal de la alimentación, aunque los supermercados e hipermercados son los minoristas con mayor cuota de 
mercado, las vinotecas están tomando cada vez más interés como establecimientos especializados.

Como se ha señalado, el presente trabajo tiene como objetivo principal analizar y comprender el proceso de compra de vino por parte de bares, restaurantes y tiendas especializadas. Para llevar a cabo la investigación se ha obtenido información primaria mediante un cuestionario estructurado aplicado de forma personal a tres tipos de compradores organizacionales minoristas en la comunidad autónoma de La Rioja. En el caso de bares y restaurantes (HORECA), se ha extraído una muestra de conveniencia de 58 establecimientos. Para las vinotecas, no se dispone de un censo exacto pero se ha contactado con un amplio número de ellas, y se dispone de información sobre 22. Para analizar la información obtenida se han utilizado distintas técnicas estadísticas (contrastes paramétricos y no paramétricos) y el programa estadístico SPSS. En el tabla 1 se recoge la ficha técnica del estudio y en el tabla 2 la descripción de la muestra.

Tabla 1. Ficha técnica de la investigación

\begin{tabular}{|c|c|}
\hline Universo & Bares, restaurantes y vinotecas \\
\hline Ámbito geográfico & La Rioja \\
\hline Población & $\begin{array}{l}\text { Establecimientos hosteleros: } 2.264 \text {; Vinotecas: no se dispone de } \\
\text { censo. }\end{array}$ \\
\hline Recogida de información & Cuestionario estructurado \\
\hline Tamaño muestral & 92 establecimientos, 80 encuestas válidas \\
\hline Fecha del trabajo de campo & Mayo 2014 - octubre 2014 \\
\hline Tipos de establecimientos & Vinotecas: $27,5 \%$; Restaurantes: $21,25 \%$; Bares: $51,25 \%$ \\
\hline
\end{tabular}

Tabla 2. Características de la muestra

\begin{tabular}{|c|l|l|l|l|}
\hline Variable & \multicolumn{2}{|c|}{ Establecimientos HORECA } & \multicolumn{2}{c|}{ Vinotecas } \\
\hline \multirow{2}{*}{ Sexo } & Hombres & $58,6 \%$ & Hombres & $59,1 \%$ \\
& Mujeres & $41,4 \%$ & Mujeres & $40,9 \%$ \\
\hline \multirow{2}{*}{ Edad } & Hasta 35 años & $36,2 \%$ & Hasta 35 años & $13,6 \%$ \\
& De 36 a 50 años & $53,4 \%$ & 36 a 50 años & $54,5 \%$ \\
& Más de 50 años & $10,4 \%$ & Más de 50 años & $31,9 \%$ \\
\hline Nivel de & Estudios primarios/Secundaria & $13,6 \%$ & Estudios primarios/Secundaria & $24,1 \%$ \\
estudios & BUP/COU/FP/Bachillerato & $63,6 \%$ & BUP/COU/FP/Bachillerato & $53,5 \%$ \\
& Con estudios universitarios & $22,8 \%$ & Con estudios universitarios & $22,4 \%$ \\
\hline
\end{tabular}




\section{RESULTADOS}

En primer lugar se analizan los cambios percibidos por los distribuidores en la oferta y la demanda de vino para, después, presentar los resultados del estudio de sus procesos de compra.

\subsection{Cambios percibidos por los intermediarios en la demanda y en la oferta de vino}

El $85 \%$ de los encuestados aseguran que se han producido cambios en la demanda de vino durante los últimos años (Gráfico 2). Entre los formulados destacan dos:

1. El crecimiento de la demanda de vino blanco (21,3\%): se asegura que se consume más que antes aunque no supera a los tintos y que a las mujeres les gustan los nuevos blancos semidulces.

2. A los clientes les gustan probar o comprar nuevos vinos (16,3\%).

Gráfico 2. Cambios percibidos en la demanda de vino en los últimos años

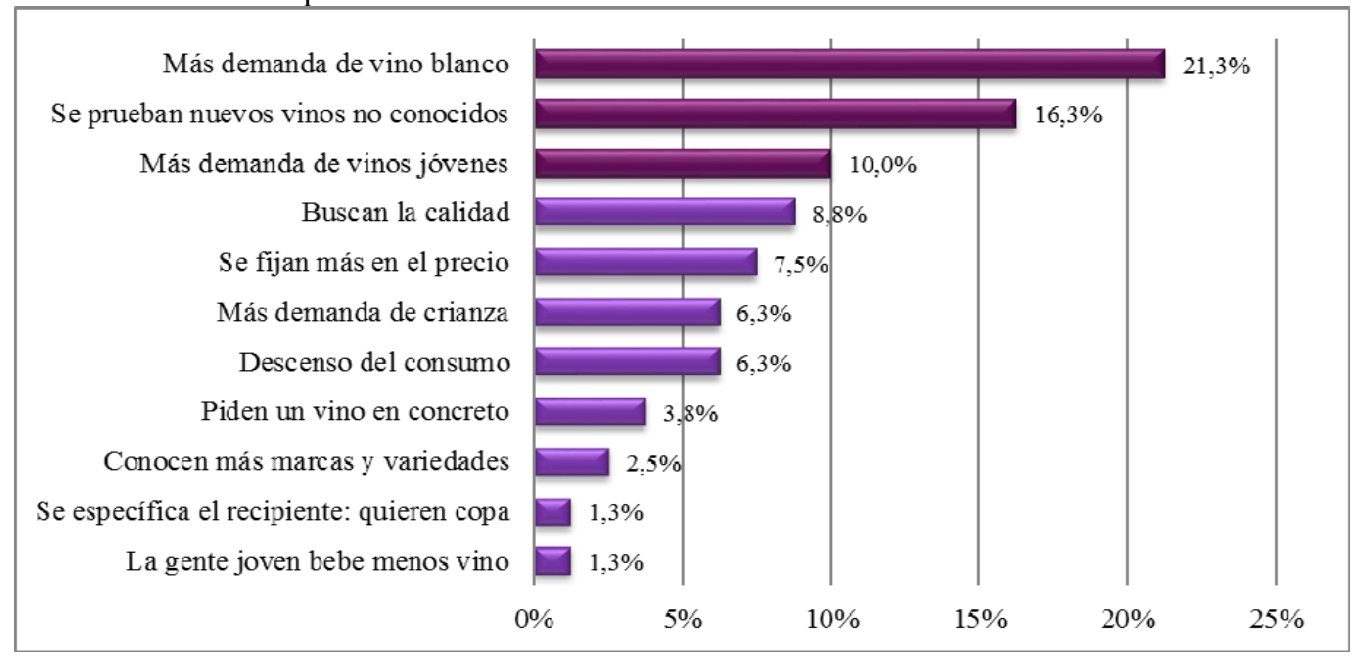

Cuando se examinan los tipos de vino que se venden por color (gráfico 3), se establecen cinco tipos: (1) tinto joven, (2) tinto crianza, (3) tintos reserva y gran reserva, (4) rosado y (5) blanco. 
Gráfico 3. Ventas de vinos diferenciados por color

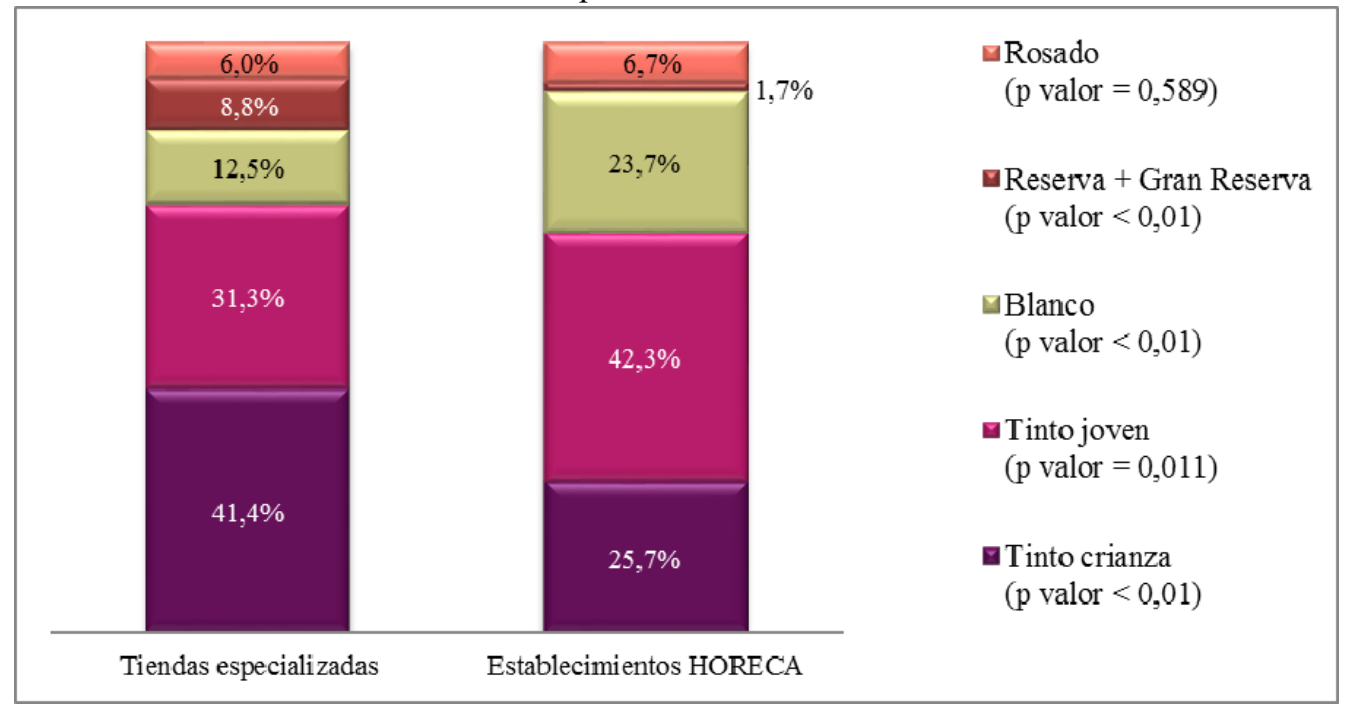

Se aprecian diferencias estadísticamente significativas entre las tiendas especializadas y los establecimientos HORECA para el vino blanco y los tintos. El vino que más se venden en los establecimientos de hostelería es el tinto joven, $42,3 \%$ en media, seguido del tinto crianza con un porcentaje medio del 25,7. En las tiendas especializadas ocurre exactamente lo opuesto: el vino que ocupa el primer puesto es el tinto crianza con un $41,4 \%$ dejando al joven en segunda posición con un $31,3 \%$ de media. El vino blanco ocupa el tercer lugar en los dos tipos de establecimientos, aunque en los establecimientos hosteleros se vende prácticamente el doble $(23,7 \%)$ que en las vinotecas $(12,5 \%)$ y casi alcanza al tinto crianza. El vino que menos se vende es el rosado con una cuota general de alrededor del $6 \%$.

Atendiendo a la denominación de origen, el Rioja se impone al resto de denominaciones tanto en tiendas especializadas $(84,4 \%)$ como en establecimientos HORECA $(87,3 \%)$.

Por otra parte y en lo que respecta a la oferta que reciben las organizaciones investigadas, un $82,5 \%$ de ellas afirman que efectivamente han detectado cambios en la oferta de vino en los últimos años. Perciben que la competencia ha aumentado y es más agresiva y, como se recoge en el gráfico 4, dicen que las visitas comerciales son más frecuentes $(13,8 \%)$ y que en éstas se ofertan mayor variedad de vinos por agente o bodega $(10 \%)$. 
Gráfico 4. Cambios percibidos en la oferta de vino en los últimos años

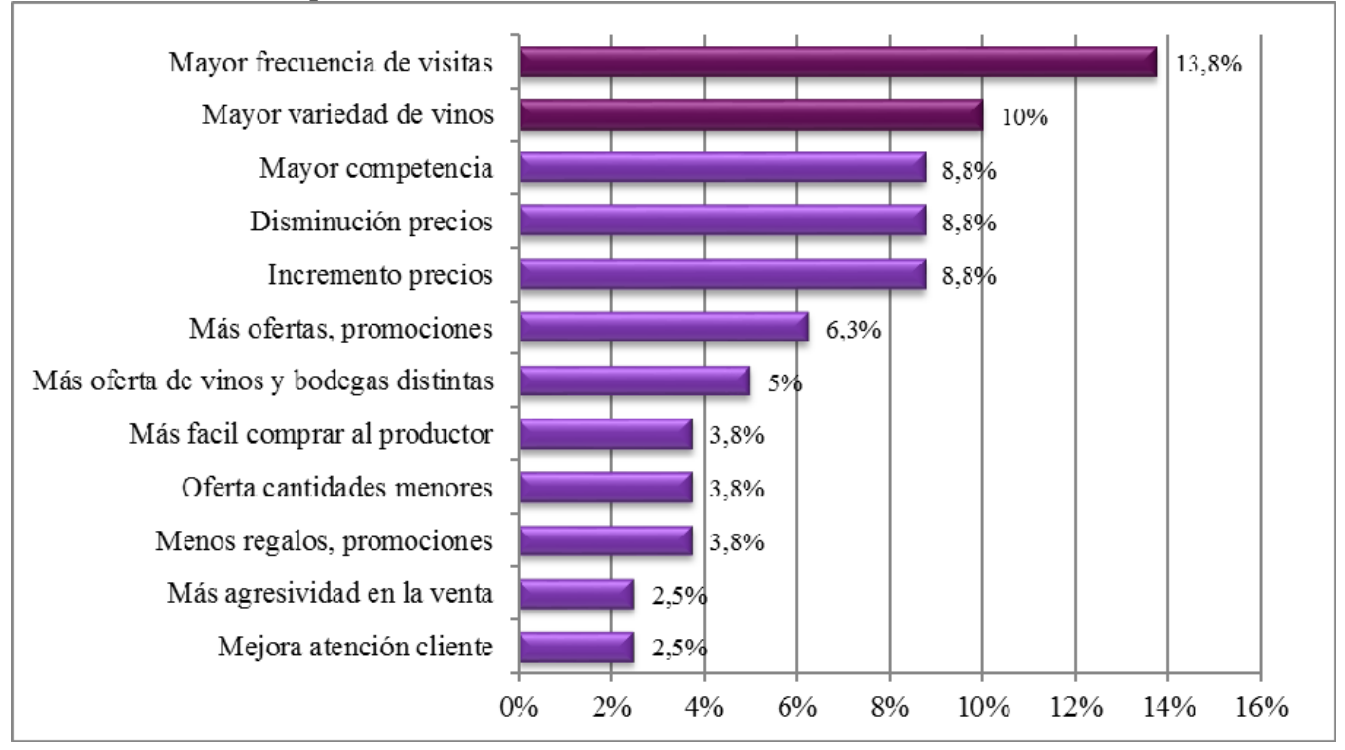

Sorprende que el $8,8 \%$ de la muestra (tiendas especializadas, bares y restaurantes) afirme que se ha producido un incremento de precios, a la vez que otro $8,8 \%$ (sólo formado por hosteleros) asegura que ha habido una disminución. Un 5\% de los encuestados han percibido una diversificación de la oferta, posiblemente relacionada con el cambio apreciado en la demanda (a los consumidores les gusta probar nuevos vinos no conocidos). Así mismo, se asegura que existe una mayor facilidad de compra directa al productor.

\subsection{Proceso de compra de vino de los minoristas}

En este apartado se van a analizar cómo las organizaciones estudiadas toman sus decisiones durante sus procesos de compra de vino.

3.2.1. Fuentes de información utilizadas para incorporar una nueva referencia de vino

La fuente de información más utilizada (gráfico 5) es el consejo, recomendación o petición de sus clientes (7,65 en una escala de 0 a 10) seguida de la opinión de familiares, amigos y conocidos $(6,04)$. 
Gráfico 5. Fuentes de información utilizadas en la búsqueda de un nuevo vino. Valoraciones medias en una escala de 0 a 10.

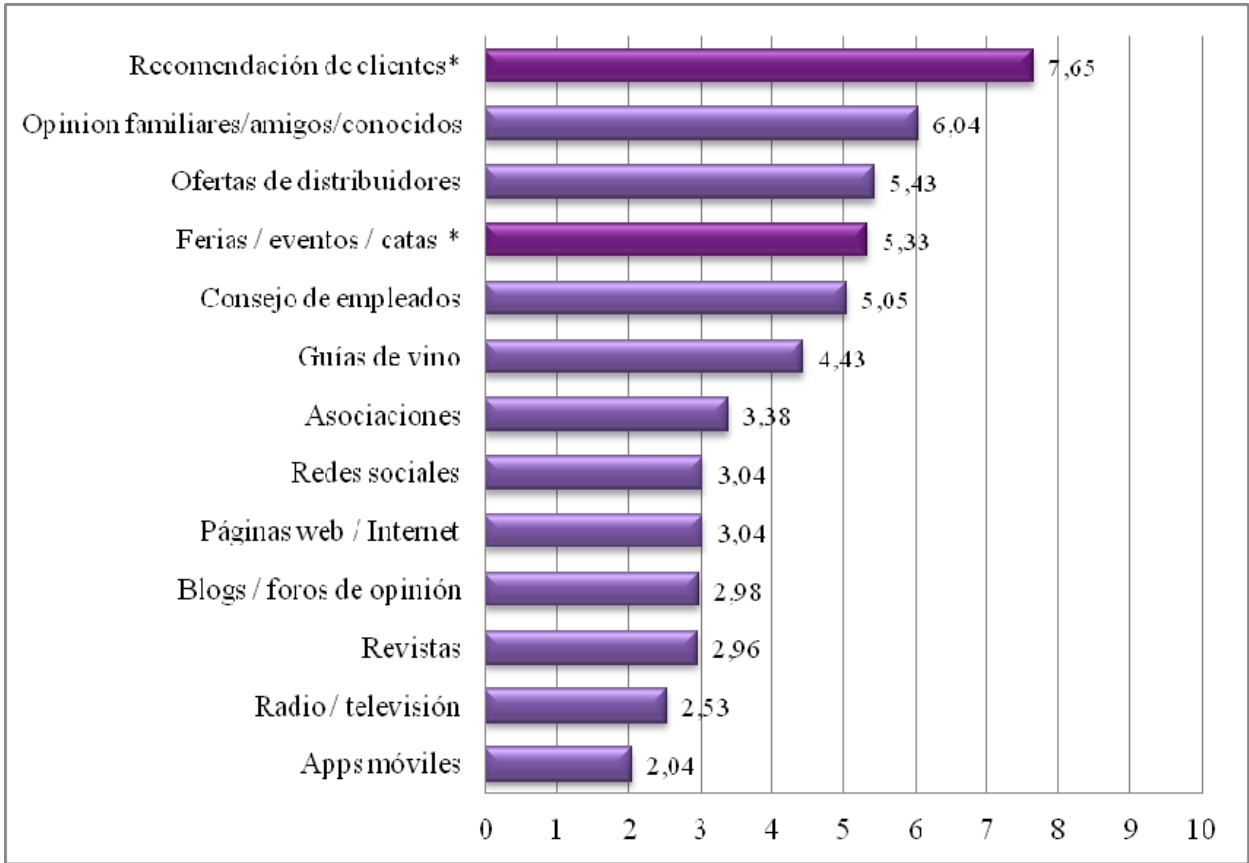

(*) Diferencias de medias estadísticamente significativas.

Cuando se comparan las valoraciones obtenidas por formatos, únicamente aparecen diferencias estadísticamente significativas para dos fuentes de información:

- Recomendación de clientes ( $\mathrm{p}$ valor $=0,069$ ): los establecimientos hosteleros confían más en la opinión de sus clientes que las tiendas especializadas.

- Ferias, eventos y catas ( $\mathrm{p}$ valor $=0,018$ ): las tiendas especializadas emplean más las ferias, los eventos o las catas como fuente de información que los establecimientos hosteleros.

\subsubsection{Aspectos valorados de un vino para incluirlo en la carta/surtido}

En una escala de 0 a 10 puntos, la calidad, la relación calidad-precio, el sabor y la denominación de origen o región de la que proviene son los aspectos más valorados (gráfico 6). Todos ellos reciben una valoración media que se encuentra entre los 8 y los 9 puntos y no se han observado diferencias estadísticamente significativas entre tipos de establecimiento. 
Gráfico 6. Aspectos valorados de un vino. Valoraciones medias en una escala de 0 a 10.

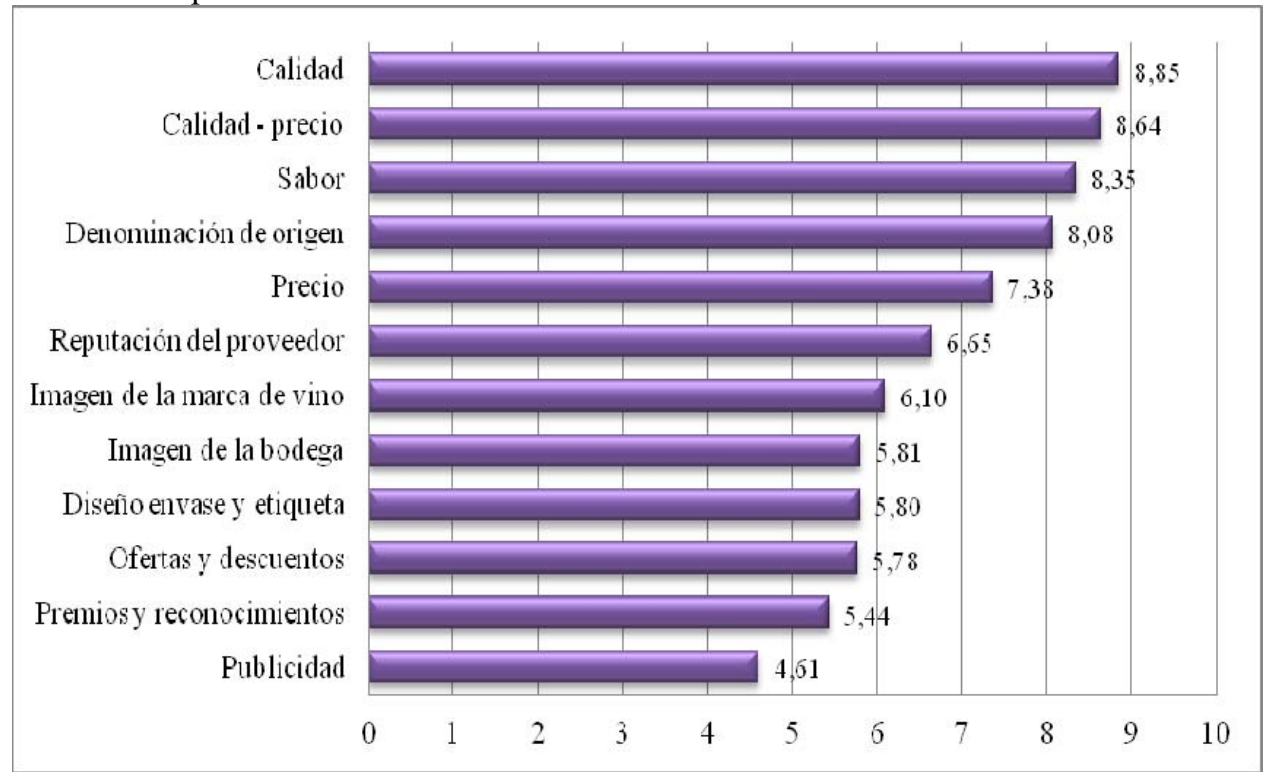

3.2.3. Motivos por los que se decide incluir un nuevo vino en la carta/surtido

Se les propuso a los compradores organizacionales cuatro posibles motivos por los que decidir incluir un nuevo vino en su carta $\mathrm{u}$ oferta para que los ordenaran según su importancia (ver resultados en tabla 3).

En general, los cuatro motivos propuestos son importantes para los intermediarios. Como primer motivo, el que más se cita es la recomendación de los clientes $(28,8 \%)$ seguido muy de cerca para innovar o actualizar $(27,5 \%)$. A la vista de los porcentajes acumulados obtenidos, haber encontrado un vino mejor y la recomendación de clientes son los motivos más mencionados considerando las tres primeras posiciones.

Tabla 3. Motivos por los que decide incluir un nuevo vino en la carta

\begin{tabular}{|l|c|c|c|c|c|c|c|c|}
\cline { 2 - 8 } \multicolumn{1}{c|}{} & $\begin{array}{c}\text { Haber encontrado } \\
\text { un vino mejor }\end{array}$ & \multicolumn{2}{c|}{$\begin{array}{c}\text { Insatisfacción vino } \\
\text { o servicio }\end{array}$} & \multicolumn{2}{c|}{$\begin{array}{c}\text { Para innovar o } \\
\text { actualizar }\end{array}$} & \multicolumn{2}{c|}{$\begin{array}{c}\text { Recomendación } \\
\text { de clientes }\end{array}$} \\
\cline { 2 - 9 } & $\%$ & $\%$ Ac & $\%$ & $\%$ Ac & $\%$ & $\%$ Ac & $\%$ & $\%$ Ac \\
\hline $\mathbf{1}^{\mathbf{0}}$ & $15 \%$ & $15 \%$ & $23,8 \%$ & $23,8 \%$ & $\mathbf{2 7 , 5 \%}$ & $27,5 \%$ & $\mathbf{2 8 , 8 \%}$ & $28,8 \%$ \\
$\mathbf{2}^{\mathbf{0}}$ & $38,8 \%$ & $53,8 \%$ & $18,8 \%$ & $42,6 \%$ & $15 \%$ & $42,5 \%$ & $27,5 \%$ & $56,3 \%$ \\
$\mathbf{3}^{\mathbf{o}}$ & $30 \%$ & $\mathbf{8 3 , 8 \%}$ & $35 \%$ & $77,6 \%$ & $21,3 \%$ & $63,8 \%$ & $23,8 \%$ & $\mathbf{8 0 , 1 \%}$ \\
\hline $\mathbf{4}^{\mathbf{0}}$ & $16,3 \%$ & $100 \%$ & $22,5 \%$ & $100 \%$ & $36,3 \%$ & $100 \%$ & $20 \%$ & $100 \%$ \\
\hline
\end{tabular}




\subsubsection{Tipos de proveedores empleados}

En el gráfico 7 se observa que los proveedores más habituales de las vinotecas, bares y restaurantes entrevistados son las bodegas $(78,8 \%)$ y los agentes o representantes (65\%). No se aprecia relación entre el tipo de establecimiento y el tipo de proveedor empleado. Los otros dos tipos de proveedores, supermercados/hipermercados y Cash\&Carry, apenas se utilizan.

\section{Gráfico 7. Proveedores de vino empleados}

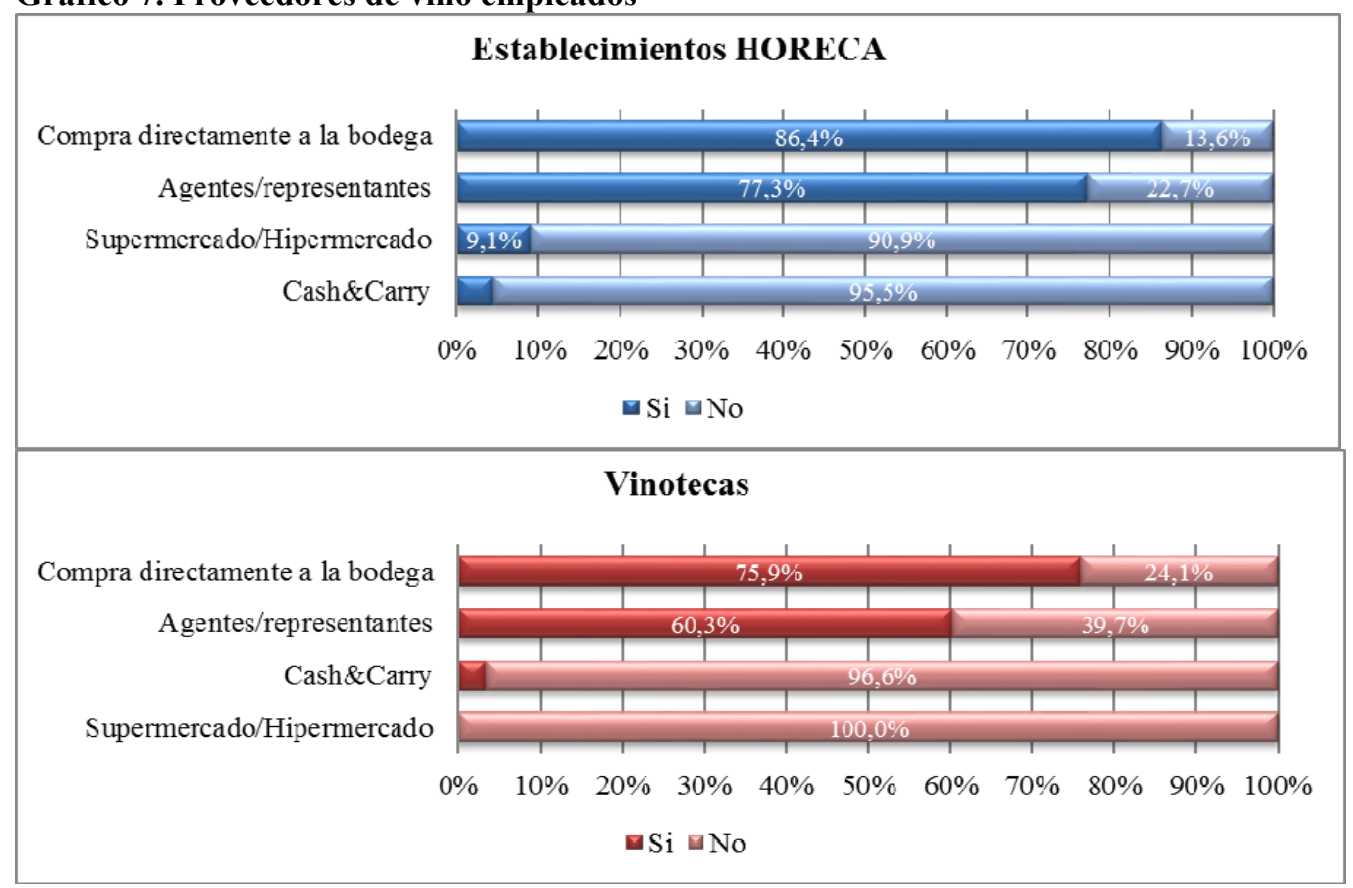

3.2.5. Aspectos valorados de un proveedor de vino

A continuación se preguntó en abierto por los aspectos que más y menos valoran los encuestados de sus principales proveedores de vino (tabla 4). En cuanto a aspectos negativos, alrededor de un $50 \%$ no tiene ninguna queja de sus proveedores. 
Tabla 4. Aspectos más y menos valorados de un proveedor de vino

\begin{tabular}{|c|c|}
\hline \multicolumn{2}{|c|}{ Aspectos más valorados de un proveedor de vino } \\
\hline Establecimientos HORECA & Tiendas especializadas \\
\hline Atención y servicio $(20,7 \%)$ & - Seriedad y formalidad $(22,7 \%)$ \\
\hline Rapidez de entrega $(17,2 \%)$ & - Calidad del producto $(18,2 \%)$ \\
\hline Seriedad y formalidad $(17,2 \%)$ & - Rapidez de entrega $(18,2 \%)$ \\
\hline \multicolumn{2}{|c|}{ Aspectos menos valorados de un proveedor de vino } \\
\hline $\begin{array}{l}\text { Inexistencia de promociones u ofertas } \\
(20,8 \%) \\
\text { Precios altos }(20,8 \%) \\
\text { Insistencia o pesadez }(20,8 \%)\end{array}$ & $\begin{array}{l}\text { - Poca atención }(27,3 \%) \\
\text { - Mal trato }(18,2 \%) \\
\text { - Impuntualidad / lentitud }(18,2 \%) \\
\text { - Irresponsabilidad }(18,2 \%)\end{array}$ \\
\hline
\end{tabular}

Gráfico 8. Valoración de las características de un proveedor de vino

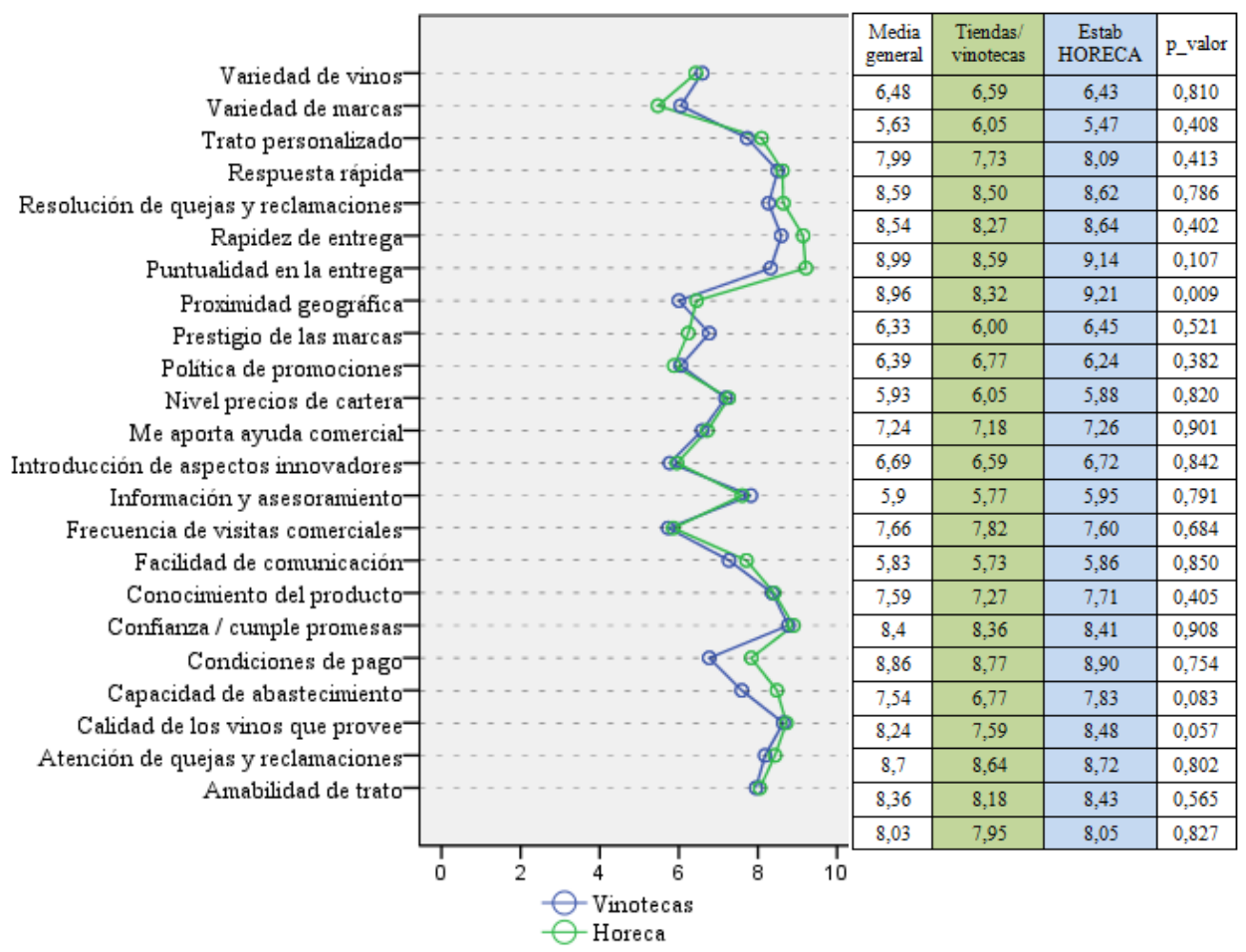

Se presentó una lista de cualidades de un proveedor de vino para que se valorasen en una escala de 0 a 10 puntos. Las respuestas emitidas en pregunta cerrada corroboran las anteriores en espontáneo; las cualidades más valoradas (gráfico 8) por encima de 8,5 puntos son: 
- $\quad$ La rapidez de entrega.

- La puntualidad en la entrega.

- La confianza que transmite.

- $\quad \mathrm{Su}$ respuesta rápida.

- La resolución de quejas y reclamaciones.

Cuando se comparan las valoraciones realizadas por los establecimientos HORECA y tiendas especializadas, únicamente aparecen diferencias estadísticamente significativas en la puntualidad en la entrega ( $\mathrm{p}$ valor $=0,009$ ) y las condiciones de pago ( $\mathrm{p}$ valor $=0,083$ ). Ambos aspectos son más valorados por los establecimientos HORECA.

\subsubsection{Medio por el que se realizan los pedidos}

La forma y la frecuencia con que los responsables de la compra de vino realizan sus pedidos se muestran en el gráfico 9.

- Los pedidos de vino se realizan mayoritariamente de forma personal y telefónica. Un 40\% combina muy frecuentemente el medio personal y el telefónico.

- El uso del correo electrónico es incipiente en los establecimientos HORECA y un $22,7 \%$ de las vinotecas lo emplean mucho. Se ha contrastado estadísticamente que su uso depende del tipo de establecimiento.

\section{Gráfico 9. Medio por el que realizan los pedidos}

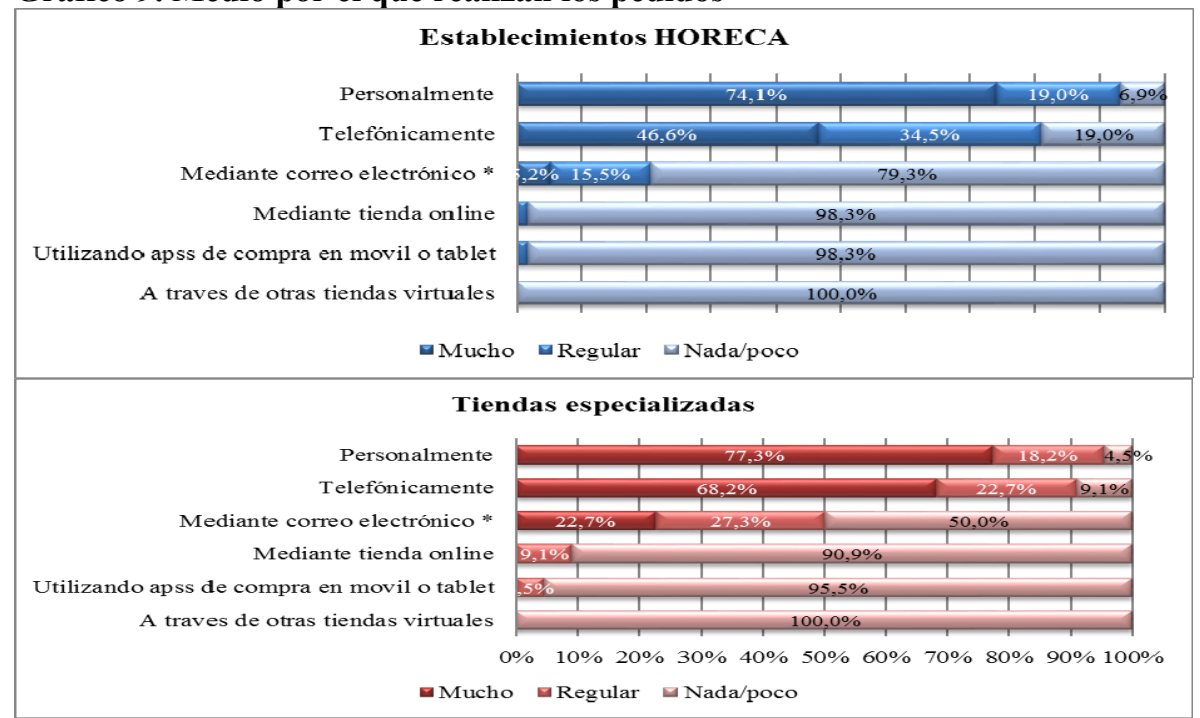

(*) Variable dependiente del tipo de establecimiento 


\subsubsection{Precio al que compran el vino los intermediarios}

Se preguntó a los encuestados a qué precio compran el vino diferenciando:

1. Precio de la botella de vino más barato.

2. Precio de la botella de vino más caro.

3. Precio de la botella de vino que más compran.

En el tabla 5 se presenta la tabla resumen de los precios a los que compran el vino los responsables de los establecimientos investigados. En el rango de precios bajos, la hostelería compra el vino a precios más bajos que en las vinotecas. No obstante la banda definida entre $1,5 €$ y $2 €$ es la más frecuente en ambos universos (50\% y $45 \%$ respectivamente).

En cuanto a las botellas de vino que más compra la muestra, el porcentaje más alto en las dos submuestras pertenece al rango que abarca desde los $1,50 €$ hasta los $3 €$. En el rango de precios más altos, las vinotecas abarcan mayores precios que los bares y restaurantes. En los establecimientos HORECA el 70,7\% de los vinos más caros se compran por debajo de $10 €$. Sin embargo en las vinotecas, el 77,3\% lo pagan por encima de $25 €$. Otro aspecto a destacar es que la más alta gama (con precios superiores a 200€) no está presente en HORECA y en tiendas especializadas alcanza el $22,7 \%$ de las compras.

\subsubsection{Fidelidad al principal proveedor de vino}

En cuanto a la fidelidad de los responsables de los establecimientos encuestados a sus proveedores de vino (gráfico 10), la mayoría:

- $\quad$ Se consideran fieles a su principal proveedor de vino $(82,5 \%)$.

- $\quad$ Recomienda a su proveedor a quien le pide consejo $(82,2 \%)$.

- Cuenta aspectos positivos sobre él a otras personas $(72,5 \%)$.

- $\quad$ En los próximos años, comprará más a ese proveedor (67,5\%).

No obstante, esa fidelidad subjetiva se reduce a la vista de las respuestas siguientes:

- A un $70 \%$ de la muestra le gusta ampliar sus relaciones comerciales con nuevos proveedores.

- Un 67,5\% no seguirá comprando al proveedor si los precios fueran más altos.

- Un $63,8 \%$ no está dispuesto a pagar un precio mayor por su servicio.

- Un $61,3 \%$ le gusta introducir nuevos vinos en la carta u oferta del establecimiento. 
Estas respuestas resultan coherentes y redundan en aspectos que ya habían surgido en el estudio como la importancia de ir renovando la oferta y la sensibilidad al precio.

Tabla 5. Precios de compra de vino

\begin{tabular}{|c|c|c|c|c|c|}
\hline & \multirow{2}{*}{$\begin{array}{l}\text { Rango de precios a } \\
\text { los que se compra la } \\
\text { botella de vino }\end{array}$} & \multicolumn{2}{|c|}{ Tiendas especializadas } & \multicolumn{2}{|c|}{ Establecimientos HORECA } \\
\hline & & Frecuencia & Frec. Acum. & Frecuencia & Frec. Acum. \\
\hline \multirow{7}{*}{ 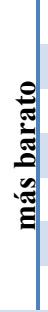 } & $0 €-1 €$ & $4,5 \%$ & $4,5 \%$ & $3,4 \%$ & $3,4 \%$ \\
\hline & $1,01 €-1,5 €$ & $4,5 \%$ & $9,1 \%$ & $13,8 \%$ & $17,2 \%$ \\
\hline & $1,51 €-2 €$ & $45,5 \%$ & $54,5 \%$ & $50,0 \%$ & $67,2 \%$ \\
\hline & $2,01 €-2,5 €$ & $18,2 \%$ & $72,7 \%$ & $17,2 \%$ & $84,5 \%$ \\
\hline & $2,51 €-3 €$ & $18,2 \%$ & $90,9 \%$ & $10,3 \%$ & $94,8 \%$ \\
\hline & $3,01 €-3,50 €$ & $4,5 \%$ & $95,5 \%$ & $3,4 \%$ & $98,3 \%$ \\
\hline & $3,51 €-4 €$ & $4,5 \%$ & $100,0 \%$ & $1,7 \%$ & $100,0 \%$ \\
\hline \multirow{5}{*}{ 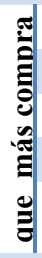 } & $1,50 €-3 €$ & $36,4 \%$ & $36,4 \%$ & $58,6 \%$ & $58,6 \%$ \\
\hline & $3,01 €-5 €$ & $18,2 \%$ & $54,5 \%$ & $27,6 \%$ & $86,2 \%$ \\
\hline & $5,01 €-7 €$ & $22,7 \%$ & $77,3 \%$ & $12,1 \%$ & $98,3 \%$ \\
\hline & $7,01 €-9 €$ & $18,2 \%$ & $95,5 \%$ & $0,0 \%$ & $98,3 \%$ \\
\hline & $9,01 €-12,30 €$ & $4,5 \%$ & $100,0 \%$ & $1,7 \%$ & $100,0 \%$ \\
\hline \multirow{7}{*}{ 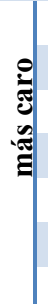 } & $3 €-6 €$ & $13,6 \%$ & $13,6 \%$ & $36,2 \%$ & $36,2 \%$ \\
\hline & $6,01 €-10 €$ & $4,5 \%$ & $18,2 \%$ & $34,5 \%$ & $70,7 \%$ \\
\hline & $10,01 €-15 €$ & $4,5 \%$ & $22,7 \%$ & $12,1 \%$ & $82,8 \%$ \\
\hline & $15,01 €-25 €$ & $0,0 \%$ & $22,7 \%$ & $8,6 \%$ & $91,4 \%$ \\
\hline & $25,01 €-50 €$ & $27,3 \%$ & $50,0 \%$ & $5,2 \%$ & $96,6 \%$ \\
\hline & $50,01 €-200 €$ & $27,3 \%$ & $77,3 \%$ & $3,4 \%$ & $100,0 \%$ \\
\hline & $200,01 €-1500 €$ & $22,7 \%$ & $100,0 \%$ & $0,0 \%$ & $100,0 \%$ \\
\hline
\end{tabular}


Gráfico 10. Escala de fidelidad a los proveedores

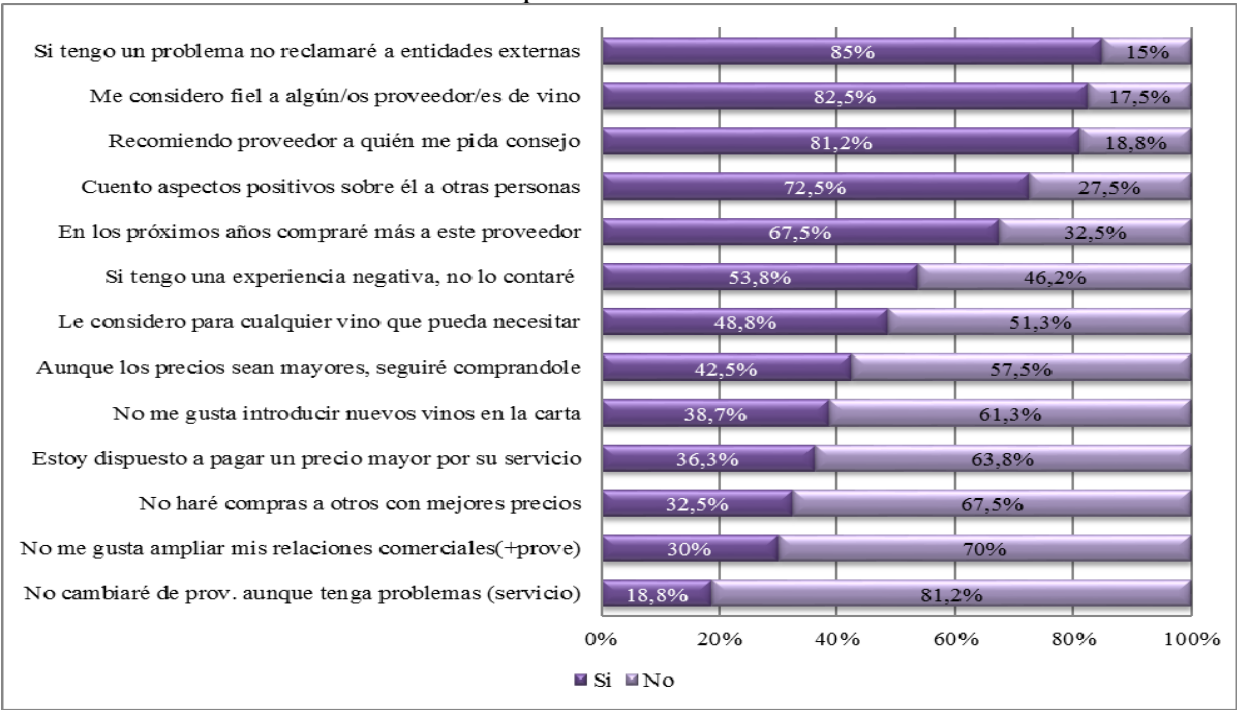

\section{RECOMENDACIONES Y CONCLUSIONES}

Los negocios de las bodegas dependen, en gran medida, de su capacidad para adecuarse a los requerimientos de sus clientes organizacionales y estos, a su vez, a los del consumidor final. Restaurantes, bares y vinotecas, como principales clientes de las pequeñas bodegas constituyen un eslabón fundamental del canal de distribución. Desde esta perspectiva y teniendo en cuenta las 8 etapas del modelo habitual de comportamiento de compra de las organizaciones, el tabla 6 recoge las conclusiones y las recomendaciones para cada una de las etapas de la compra organizacional de un vino.

Tabla 6. Conclusiones y recomendaciones sobre la compra organizacional de vino

\begin{tabular}{l|l}
\hline & $\begin{array}{l}\text { El proceso de compra organizacional se inicia con el reconocimiento de un } \\
\text { problema o necesidad debido a estímulos internos o externos. } \\
\text { Los compradores organizacionales (bares, restaurantes y vinotecas) destacan los } \\
\text { motivos externos como claves para incluir un nuevo vino en su carta. Los más } \\
\text { mencionados fueron: } \\
\begin{array}{l}\text { Etapa 1 } \\
\text { Reconocimiento } \\
\text { del problema o } \\
\text { necesidad. }\end{array}\end{array}$ \\
& $\begin{array}{l}\text { Recomendación o sugerencia de los propios clientes. Los } \\
\text { requerimientos de los consumidores de probar nuevos productos } \\
\text { presionan a los minoristas a incorporar nuevos vinos en su oferta. }\end{array}$ \\
& $\begin{array}{l}\text { Haber encontrado un vino mejor. } \\
\text { En esta primera etapa, el papel de las bodegas consiste en estimular el } \\
\text { reconocimiento de la necesidad enviando información de sus nuevos vinos a } \\
\text { través de sus representantes o directamente, por ejemplo, mediante correo }\end{array}$ \\
\hline
\end{tabular}




\begin{tabular}{|c|c|}
\hline & electrónico. \\
\hline $\begin{array}{l}\text { Etapa } 2 \text { y } 3 \\
\text { Descripción } \\
\text { general de la } \\
\text { necesidad y de } \\
\text { las } \\
\text { especificaciones } \\
\text { del producto. }\end{array}$ & $\begin{array}{l}\text { El comprador organizacional determina las características y cantidad de unidades } \\
\text { que necesita. En el caso de bares, restaurantes y vinotecas señalan la calidad, la } \\
\text { relación calidad precio, el sabor y la denominación de origen o zona de } \\
\text { procedencia como los aspectos que tienen más en cuenta a la hora de elegir un } \\
\text { vino para incluirlo en la oferta de un establecimiento. Más en concreto, cabe } \\
\text { destacar: } \\
\text { - La Denominación de Origen Calificada Rioja se impone al resto tanto en } \\
\text { tiendas especializadas (84,4\%) como en establecimientos HORECA } \\
\text { ( } 87,3 \%) \text {. } \\
\text { Por colores se impone el tinto. El vino blanco, aunque con una presencia } \\
\text { creciente, se sitúa en tercer lugar en los dos canales. } \\
\text { Cuando se habla calidad del vino vendido, surgen diferencias: en las tiendas } \\
\text { especializadas se vende mayor calidad ya que el vino más demandado es el } \\
\text { tinto crianza, seguido del tinto joven. En los bares y restaurantes ocurre } \\
\text { exactamente lo contrario. } \\
\text { Las bodegas se interesarán por dar soluciones y propuestas alternativas aportando } \\
\text { información sobre sus productos y relativa a los aspectos anteriores. }\end{array}$ \\
\hline $\begin{array}{l}\text { Etapa } 4 \\
\text { Búsqueda de } \\
\text { proveedores. }\end{array}$ & $\begin{array}{l}\text { Cuando una organización minorista busca un proveedor para un nuevo producto, } \\
\text { las bodegas deben estar allí donde sus clientes organizacionales buscan } \\
\text { información. } \\
\text { Las fuentes de información más empleadas para seleccionar un nuevo vino son } \\
\text { las de tipo personal: en primer lugar la recomendación de los clientes y en } \\
\text { segundo lugar la opinión de familiares, amigos y conocidos. También destaca la } \\
\text { consideración de ferias, eventos y catas por parte de las vinotecas. } \\
\text { Sorprende que las páginas web e internet apenas se utilicen como fuente de } \\
\text { información. } \\
\text { Los minoristas utilizan dos tipos básicos de aprovisionamiento: compra directa a } \\
\text { bodega y compra a agentes comerciales o representantes. El } 36,2 \% \text { de los } \\
\text { establecimientos hosteleros encuestados y el } 63,6 \% \text { de las vinotecas emplean } \\
\text { ambos tipos de proveedores simultáneamente. }\end{array}$ \\
\hline
\end{tabular}

\section{Etapa 5}

Solicitud de propuestas.

\section{Etapa 6}

Selección de proveedores.
Cuando el comprador organizacional pide propuestas, la bodega debe realizarlas en función de los beneficios buscados por el primero.

Tanto las bodegas como las organizaciones que operan como intermediarios han de seguir muy de cerca la evolución de los hábitos del consumidor final. En este sentido, un $85 \%$ de los minoristas han percibido cambios en la demanda de los últimos años siendo dos los más repetidos: creciente demanda de vino blanco $(21,3 \%)$ y creciente afán de los consumidores por probar vinos nuevos $(16,3 \%)$.

En la selección de un proveedor, las bodegas deben conocer los criterios de valoración de sus clientes minoristas para conseguir ser elegidas. Los minoristas señalan, de forma espontánea, que los aspectos más valorados de un proveedor son:

- Atención y servicio, seriedad y formalidad.

- $\quad$ Rapidez de entrega

- Disponibilidad

\section{No les gusta:}

- La insistencia o pesadez

- Los precios altos y ausencia de promociones y ofertas.

Cuando se les presenta una escala, se corrobora que las cualidades mejor 


\section{Etapa 7}

Especificación de la rutina de pedido.

\section{Etapa 8}

Revisión de resultados. valoradas de un proveedor son la rapidez de entrega, la puntualidad, la confianza y la calidad de los vinos que provee.

En cuanto al precio, tanto la hostelería como las vinotecas, el vino que más compran y el vino que compran más barato se comercializan a precios similares. También se ha observado como en vinotecas se compran vinos de alta gama que no están presentes en HORECA.

La forma más habitual de realizar los pedidos es personalmente o por teléfono. Ambos medios se utilizan simultáneamente en un $40 \%$ de los casos. Los minoristas apenas emplean las nuevas tecnologías.

Más de la mitad de los minoristas aseguran que no tiene ninguna queja sobre su principal suministrador de vino. Cuando las tienen, (1) en establecimientos HORECA se quejan de la ausencia de promociones y ofertas y de la excesiva insistencia de visitas comerciales; (2) en tiendas especializadas, se reclama más atención y mejor trato.

Más del $80 \%$ de los encuestado se consideran fieles a algún/os proveedor/es de vino. Por otro lado, el $81,2 \%$ afirma que si tuviera algún problema grave con su servicio rompería la relación con el mismo y buscaría otro, aspecto relacionado con que la rapidez de entrega, respuesta rápida, puntualidad, disponibilidad y atención son factores muy influyentes en la opinión sobre el distribuidor de vino. Además, a un $70 \%$ de la muestra le gusta ampliar sus relaciones comerciales con nuevos proveedores e introducir nuevos vinos en la carta $u$ oferta del establecimiento $(61,3 \%)$.

Como conclusiones generales, se observa una clara orientación al cliente por parte de vinotecas y establecimientos HORECA. Se trata de un cliente final que conoce y pide marcas concretas, al que le gusta probar nuevas propuestas y a una buena relación calidad-precio. En consecuencia, las bodegas han de estar atentas a la demanda final y a su evolución así como cultivar la fidelidad de sus clientes minoristas:

- Ofertando novedades de vino en línea con las demandas de los consumidores finales.

- Haciendo un número de visitas adecuado, sin presionar y con un servicio rápido y puntual.

- Utilizando políticas de precios dinámicas para satisfacer a un cliente que, tras la crisis, es más sensible al precio y ávido de promociones.

- Ganando la confianza de sus clientes aportándoles valor y ayudándoles a satisfacer al cliente final.

- Además y puesto que se ha detectado un escaso uso de las nuevas tecnologías por parte de los distribuidores minoristas investigados, también podrían facilitarles su acceso y demostrarles su utilidad y rentabilidad para realizar ciertas funciones, sin menoscabo de la relación personal.

En definitiva, las bodegas deben gestionar su presencia activa en cada una de las 
etapas del proceso de compra de sus clientes: desde el reconocimiento de la necesidad, la búsqueda de información, la elección de proveedor, la comparación de precios, la decisión de compra y el pago, hasta los servicios postventa. Y todo ello, desde los canales offline a los online porque la demanda final se mueve y comparte ambos universos.

\section{Agradecimientos}

Este estudio se integra dentro del proyecto titulado "Elaboración de vinos espumosos de calidad a partir de variedades tintas. Estudio de diferentes técnicas vitivinícolas" (número de referencia RTA2012-00092-C02) cofinanciado por el Instituto Nacional de Investigación y Tecnología Agraria y Alimentaria (INIA) y fondos FEDER.

\section{BIBLIOGRAFIA}

BARCO, E., NAVARRO, M. C. y PINILLOS, M. (2007). "Estrategias de diferenciación versus estrategias de tamaño: el caso de la DOCa Rioja", Revista Española De Estudios Agrosociales y Pesqueros, Vol. 215-216, p. 1345.

BROWN, B. P., ZABLAH, A. R., BELLENGER, D. N. y DONTHU, N. (2012). "What factors influence buying center brand sensitivity?", Industrial Marketing Management, Vol. 41, p. 508-520.

FORTIN, P. A. y BRENT RITCHIE, J. (1980). "Influence structure in organizational buying behavior", Journal of Business Research, Vol. 8, nº 3 . 277-299.

FUENTES-LOMBARDO, G., FERNÁNDEZ-ORTIZ, R. y CANO-RUBIO, M. (2011). "Intangibles en la internacionalización de las bodegas españolas: Percepción directiva y comparada entre empresas familiares y no familiares", Intangible Capital, Vol. 7, nº 2, p. 428-473.

HONNALLI, S. H. (2011). "An overview of industrial Marketing", Advances in Management, Vol. 4 (11).

JOHNSTON, W. y BONOMA, T. (1981). "The buying center: structure and interaction patterns", Journal of Marketing, Vol. 45, p. 143-56.

JOHNSTON, W. y LEWIN, J. (1996). "Organizational buying behavior: Toward an integrative framework", Journal of Business Research, Vol. 35, $\mathrm{n}^{\circ} 1$, p. 115.

JOHNSTON, W. y SPEKMAN, R. (1987). "Industrial buying behavior: where we are and where we need to go", Research in Consumer Behavior, Vol. 1, p. 83111. 
KOTLER, P. y KELLER, K.L. (2012). Dirección de marketing. Ed. Pearson, 14a edición, p. 182-210.

KRALJIC, P. (1993). Purchasing Must Become Supply Management. Harvard Business Review, p. 109-117.

LEWIN, J. y DONTHU, N. (2005). "The influence of purchase situation on buying center structure and involvement: A select meta-analysis of organizational buying behavior research", Journal of Business Research, Vol. 58, n¹0, p. 1381.

MANRIQUE MOLINA, L. F. (2014). "Comportamiento de compra organizacional, una exploración del estado actual". Revista Virtual Universidad Católica del Norte, Vol. 4(43), p.113-130.

MORIARTY, R. T. y SPEKMAN, R. E. (1984). "An empirical investigation of the information sources used during the industrial buying process", Journal of Marketing Research, Vol. 21, $\mathrm{n}^{\circ}$ 2, 137-147.

NIELSEN (2014). "Informe anual del mercado de vinos con D.O.Ca Rioja. Mercado español: canales de alimentación y hostelería en 2013". Edita Consejo Regulador de D.O.Ca Rioja.

ROBINSON, P. J., FARIS, C. W. y WIND, Y. (1967). Industrial Buying and Creative Marketing, Boston: Allyn \& Bacon.

RONCHETTO, J., HUNT, M. y REINGEN, P. (1989). "Embedded influence patterns in organizational buying systems", Journal of Marketing, Vol. 53, $\mathrm{n}^{\circ}$ 4, p. 51-62.

SHETH, J. N. (1973). "A model of industrial buyer behavior", Journal of Marketing, Vol. 37, Vol. 37, $\mathrm{n}^{\mathrm{o}}$ 4, p. 50-56.

SHETH, J.N. (1996). "Organizational buying behavior: past performance and future expectations", Journal of Business \& Industrial Marketing, Vol. 11, Iss: 3/4, p. 7-24.

WEBSTER, F. E. y WIND, Y. (1972a). "A general model for understanding organizational buying behavior", Journal of Marketing, Vol. 36, $\mathrm{n}^{\circ}$ 2, p. 12 19.

WEBSTER, F. E. y WIND, Y. (1972b). Organizational Buying Behavior. N.J.: Englewood Cliffs.

WILSON, D. (1995). "An integrated model of buyer-seller relationships", Journal of the Academy of Marketing Science, Vol. 23, pp 335-45.

WILSON, E., LILIEN, G. y WILSON, D. (1991). "Developing and testing a contingency paradigm of group choice in organizational buying", Journal of Marketing Research, Vol. 28, p. 452-66.

WIND, Y. (2006). "Blurring the lines: is there a need to rethink industrial marketing?", Journal of Business \& Industrial Marketing, Vol. 21(7), p. 474481. 